\title{
Keratin Variants Are Overrepresented in Primary Biliary Cirrhosis and Associate with Disease Severity
}

\author{
Bihui Zhong, ${ }^{1,2 *}$ Pavel Strnad, ${ }^{2,3 *}$ Carlo Selmi, ${ }^{4,5}$ Pietro Invernizzi, ${ }^{4}$ Guo-Zhong Tao,${ }^{2}$ Angela Caleffi, ${ }^{6}$ Minhu Chen, ${ }^{1}$ \\ Ilaria Bianchi, ${ }^{4,5}$ Mauro Podda, ${ }^{4,5}$ Antonello Pietrangelo, ${ }^{6}$ M. Eric Gershwin, ${ }^{7}$ and M. Bishr Omary ${ }^{8}$
}

Keratins (K) 8 and 18 variants predispose carriers to the development of end-stage liver disease and patients with chronic hepatitis $\mathrm{C}$ to disease progression. Hepatocytes express $\mathrm{K} 8 / \mathrm{K} 18$, whereas biliary epithelia express K8/K18/K19. K8-null mice, which are predisposed to liver injury, spontaneously develop anti-mitochondrial antibodies (AMA) and have altered hepatocyte mitochondrial size and function. There is no known association of K19 with human disease and no known association of K8/K18/K19 with human autoimmune liver disease. We tested the hypothesis that K8/K18/K19 variants associate with primary biliary cirrhosis (PBC), an autoimmune cholestatic liver disease characterized by the presence of serum AMA. In doing so, we analyzed the entire exonic regions of K8/K18/K19 in 201 Italian patients and 200 control blood bank donors. Five disease-associated keratin heterozygous variants were identified in patients versus controls (K8 G62C/R341H/V380I, K18 R411H, and K19 G17S). Four variants were novel and included K19 G17S/V229M/N184N and K18 R411H. Overall, heterozygous diseaseassociated keratin variants were found in 17 of $201(8.5 \%)$ PBC patients and 4 of $200(2 \%)$ blood bank donors $(P<0.004$, odds ratio $=4.53,95 \%$ confidence interval $=1.5-13.7)$. Of the K19 variants, K19 G17S was found in three patients but not in controls and all K8 R341H (eight patients and three controls) associated with concurrent presence of the previously described intronic K8 IVS7+10delC deletion. Notably, keratin variants associated with disease severity (12.4\% variants in Ludwig stage III/IV versus $4.2 \%$ in stages $\mathrm{I} / \mathrm{II} ; \boldsymbol{P}<0.04$, odds ratio $=3.25$, $95 \%$ confidence interval $=1.02-10.40$ ), but not with the presence of AMA. Conclusion: K8/K18/ $\mathrm{K} 19$ variants are overrepresented in Italian $\mathrm{PBC}$ patients and associate with liver disease progression. Therefore, we hypothesize that $\mathrm{K} 8 / \mathrm{K} 18 / \mathrm{K} 19$ variants may serve as genetic modifiers in PBC. (Hepatology 2009;50:546-554.)

$\mathrm{P}$ rimary biliary cirrhosis $(\mathrm{PBC})$ is a slowly progressive autoimmune liver disease characterized by periportal intrahepatic inflammation and immunemediated destruction of the small bile ducts. The loss of

cholangiocytes leads to decreased bile secretion and retention of toxic substances and may result in cirrhosis and eventually liver failure. ${ }^{1,2} \mathrm{PBC}$ is found primarily in female patients, with middle-age onset, ${ }^{1,3}$ with an estimated

\footnotetext{
Abbreviations: $A M A$, anti-mitochondrial antibody, DHPLC, denaturing high-performance liquid chromatography; h, human; IFs, intermediate filaments; K, keratin protein; $m$, mouse; $P B C$, primary biliary cirrhosis; $P C R$, polymerase chain reaction.

From the ${ }^{1}$ Division of Gastroenterology, First Affliated Hospital of Sun Yat-sen University, Guangzhou, China; ${ }^{2}$ Palo Alto VA Medical Center and Stanford University School of Medicine, Palo Alto, CA; the ${ }^{3}$ Department of Internal Medicine I, University Medical Center Ulm, Ulm, Germany; the ${ }^{4}$ Division of Internal Medicine and Hepatobiliary Immunopathology Unit, Rozzano, Italy; the ${ }^{5}$ University of Milan, Rozzano, Italy; the ${ }^{6}$ Center for Hemochromatosis, Department of Internal Medicine, University of Modena and Reggio Emilia, Modena, Italy; and the 7 Division of Rheumatology, Allergy, and Clinical Immunology, University of California Davis, Davis, CA; and the ${ }^{8}$ Department of Molecular \& Integrative Physiology, University of Michigan School of Medicine, Ann Arbor, MI.

*These authors contributed equally to this work.

Supported by the Department of Veterans Affairs and National Institutes of Health (NIH) grant DK52951 (M.B.O.); NIH Center grant DK56339 to Stanford University; NIH grant DK39588 (M.E.G); Guangdong province international cooperative grant 2004 B50301015 (B.Z.); and the Emmy Noether program of German Research Foundation (grant STR 1095/2-1 to P.S.).

Received January 27, 2009; accepted April 16, 2009.

Address correspondence to: Bishr Omary, University of Michigan, School of Medicine, Department of Molecular \& Integrative Physiology, 7744 Medical Science II, 1301 E. Catherine Street, Ann Arbor, MI 48109; or Bihui Zhong, Division of Gastroenterology, First Affiliated Hospital of Sun Yat-sen University, Guangzhou 510080 , PR China.

Copyright (C) 2009 by the American Association for the Study of Liver Diseases.

Published online in Wiley InterScience (www.interscience.wiley.com).

DOI 10.1002/hep.23041

Potential conflict of interest: Nothing to report.

Additional Supporting Information may be found in the online version of this article.
} 
prevalence in the United States and England of 20 to 40 per 100,000. ${ }^{4}$ PBC causes a significant economic burden and a mortality rate in the United States of 0.24 per $100,000.5,6$ The cause of PBC is unclear, but it is an autoimmune disease that appears to be related to environmental factors, such as chemicals and bacteria, acting on genetically susceptible individuals. ${ }^{1,3}$ The importance of genetic factors for the susceptibility to $\mathrm{PBC}$ is evidenced by the increased incidence among first-degree relatives of affected individuals, ${ }^{7}$ the high concordance rate among monozygotic twins, ${ }^{8}$ and the common coexistence with other autoimmune diseases. ${ }^{9}$ However, no definitive genetic associations with $\mathrm{PBC}$ onset or progression are established, though several genetic factors conferring susceptibility to PBC have been investigated. ${ }^{1,10-12}$

Keratins $(\mathrm{K})$ are the largest subgroup among the intermediate filament (IF) family of cytoskeletal proteins. All IF proteins, including keratins, have a characteristic "rod" domain that is flanked by N-terminal "head" and C-terminal "tail" domains. The 37 known human keratin functional genes, which exclude hair keratins, are grouped into relatively basic type II (K1-K5/K6a-K6c/K7/K8/K71-K80) and relatively acidic type I (K9/K10/K12-K20/K23-K28) keratins. ${ }^{13,14}$ Epithelial cells express at least one type I and one type II keratin $^{13}$ in an epithelial cell-specific manner. For example, "simple" (single-layered secretory or absorptive) epithelia as found in the liver, pancreas, and intestine express primarily K8/K18 (with variable levels of K7/K19/K20), whereas keratinocytes express primarily $\mathrm{K} 5 / \mathrm{K} 14$ basally and K1/K10 suprabasally. ${ }^{15}$ In the liver, adult hepatocytes express K8/K18 exclusively, whereas biliary epithelia express K7/K8/K18/K19. ${ }^{15}$ Simple epithelial keratins carry out a number of functions, including cytoprotection (for example, from apoptotic injury); protein targeting and synthesis; and modulation of mitochondrial subcellular organization, size, and function. ${ }^{15}$

The use of transgenic animals as potential human disease models has demonstrated the importance of keratin variants in various epithelial disorders, including those involving the epidermis and liver. For example, K8-null mice manifest $94 \%$ embryo lethality with extensive liver hemorrhage and susceptibility to liver injury. ${ }^{16,17}$ Similarly, transgenic mice that express K18 Arg89 $\rightarrow$ Cys develop mild chronic hepatitis with a marked predisposition to liver injury. ${ }^{18}$ These animal model data led to human studies that demonstrated that $\mathrm{K} 8$ and $\mathrm{K} 18$ variants are overrepresented in patients with various types of endstage liver disease (12.4\% of 467 explants) as compared with blood bank controls $(3.7 \%$ of 349 donors, $P<$ 0.0001). ${ }^{19,20}$ In addition, patients with chronic hepatitis $\mathrm{C}$ virus infection have a $\mathrm{K} 8$ variant frequency that increases in a significant manner with fibrosis progression. ${ }^{21}$
Transgenic mice that express either of the two major human liver disease-associated $\mathrm{K} 8$ variants (K8 G62 $\mathrm{C}^{22}$ and $\mathrm{R} 341 \mathrm{H}$ [unpublished observations]) support the human genetic data because both mouse models are predisposed to liver injury when challenged with hepatotoxins.

Several observations support the hypothesis that K8/K18/K19 variants may predispose to autoimmune liver disease. For example, 12 of the 467 previously analyzed cases of end-stage liver disease were patients with $\mathrm{PBC}$ in whom K8/K18 variants were found in three patients $(25 \%) .{ }^{20}$ In addition, serum anti-mitochondrial antibodies (AMA) are found in K8-null mice in association with alterations in mitochondrial size, organization, and function (unpublished observations). These observations led us to examine the presence of keratin variants in a well-defined cohort of Italian patients with $\mathrm{PBC}$ as compared with blood bank controls. In addition to K8/K18, we also examined the potential presence of genetic K19 variants given $\mathrm{K} 19$ expression in biliary epithelia. ${ }^{15} \mathrm{Be}-$ cause the relative expression of simple epithelial keratins in biliary epithelia has not been examined, we also compared the expression of these keratins in mouse common bile duct and liver with the gallbladder and intestine. Of note, the association of K19 variants has not been described in any human disease, although few K19 polymorphisms have been reported in controls and patients with inflammatory bowel disease. ${ }^{23}$

\section{Patients and Methods}

Mice. One-month-old female FVB/N mice were used to compare keratin expression in several digestive tissues. Mice were euthanized by $\mathrm{CO}_{2}$ inhalation followed by removal of the common bile duct, liver, gallbladder, and small intestine. Tissues were snap-frozen in liquid $\mathrm{N}_{2}$ for subsequent biochemical analysis. All animals received humane care, and their use was approved by the institutional animal care committee.

High Salt Extraction and Western Blot Analysis. Enriched keratin fractions were obtained by high salt extraction as described. ${ }^{24}$ The samples were separated by sodium dodecyl sulfate polyacrylamide gel electrophoresis followed either by Coomassie blue staining or by transfer to membranes for immunoblotting. Keratins were detected using enhanced chemiluminescence (PerkinElmer) and the primary antibodies: rabbit anti-human (h)/mouse (m) K18 (antibody 4668) 25; rat anti-mK19 (Troma-III; Developmental Hybridoma Bank); mouse anti-h/mK7 (RCR105, Progen Biotechnik GmbH); anti-mK20 (K20.8, Neo-Markers).

PBC Patient and Control Cohorts. Genomic DNA was isolated from well-characterized 209 Italian patients 
with PBC (Milan, Italy) and from 200 Italian blood bank donors (Modena, Italy). The diagnosis of PBC was based on internationally accepted standards, ${ }^{1}$ including the presence of two of three criteria: (1) positive serum AMA, (2) serum alkaline phosphatase more than two times normal for 6 months or longer, or (3) a compatible liver histology. In subjects without detectable AMA, the patients had to fulfill both remaining criteria to be enrolled. Serum AMA were determined using indirect immunofluorescence and titers 1:40 or greater were considered positive. Patients were considered to be positive for antinuclear antibodies when they displayed elevated titers of anti-sp100 or anti-gp210 antibodies or positive indirect immunofluorescence. Liver biopsy staging was based on Ludwig's criteria. ${ }^{26}$ The absence of liver fibrosis was defined as early-stage PBC (Ludwig stages I-II), and the advanced stage was defined as the evidence of liver fibrosis or cirrhosis (Ludwig stages III-IV) or those who had undergone liver transplantation or a cirrhosis-related complication. The Mayo score was employed to evaluate the prognosis of liver disease at the time of DNA sampling. ${ }^{27}$ Patients positive for serum hepatitis B surface antigen, hepatitis $\mathrm{C}$ virus antibody, with a history of alcohol abuse within 1 year of diagnosis, or bile duct obstruction (based on ultrasound, computer tomography, or endoscopic evaluation) were excluded.

Human Genomic DNA Samples and Polymerase Chain Reaction Analysis. Genomic DNA was isolated from peripheral whole blood using DNeasy tissue kit (Qiagen Inc). All the exonic regions and exonic-intronic boundaries of K8/K18/K19 were analyzed using previously described primers. ${ }^{21,23} \mathrm{DNA}$ fragments were amplified with a Touchdown polymerase chain reaction (PCR) protocol using T-TaqTM Polymerase (Transgenomic) to obtain high amplification specificity. ${ }^{28}$ The PCR reactions were performed with a Gene Amp PCR system 9700 (Applied Biosystems Inc.).

\section{Denaturing High-Performance Liquid Chromatog-} raphy. The human samples were screened for the presence of K8/K18/K19 variants via denaturing high-performance liquid chromatography (DHPLC) using WAVE Fragment Analysis System (Transgenomic). ${ }^{21,28}$ Samples with a "shifted" elution pattern were purified using a PCR purification kit (Qiagen Inc.) and sequenced bidirectionally to confirm the presence of a mutation. The messenger RNA sequences of K8 (NM002273), K18 (NM000224), and K19 (NM002276) were used to localize the coding variants, whereas genomic sequences (hKRT8 [M34482], hKRT18 [AF179904], and hKRT19 [AF202321]) were employed for noncoding variants.

Statistical Analysis. For continuous variables, the two-tailed $t$ test was used to compare two groups together,

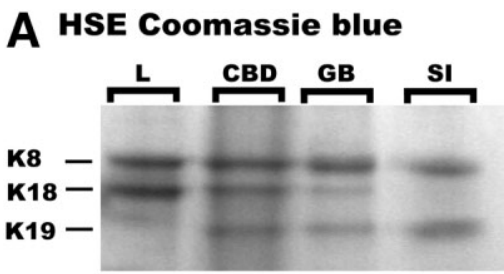

B Western blot

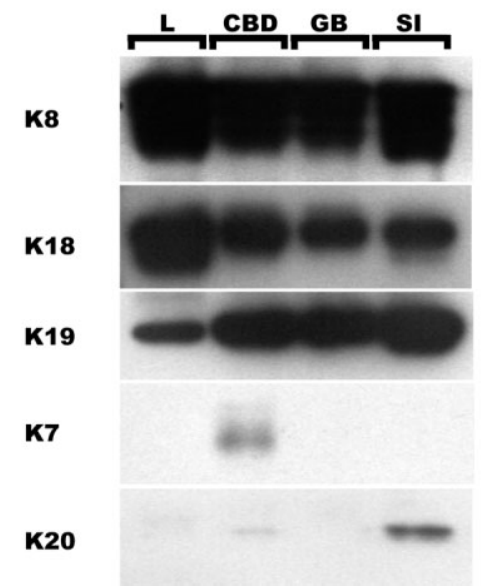

Fig. 1. Expression of keratins in the liver, common bile duct, gallbladder, and small intestine. Keratin-enriched fractions were prepared by high salt extraction from liver (L), common bile duct (CBD), gallbladder (GB), and small intestine (SI) then separated by sodium dodecyl sulfate polyacrylamide gel electrophoresis. The organs were resected from 1-month-old female FVB/N mice. Gels were stained with Coomassie blue (A) or transferred to membranes and immunoblotted with antibodies to the indicated keratin species (B).

whereas the Kruskal-Wallis nonparametric one-way analysis of variance was used to compare more than two groups. K8/K18/K19 variant frequencies in patient and control groups were compared with the two-tailed Fisher exact probability test. $P<0.05$ was considered statistically significant. Statistical analysis was performed using SAS Institute system (version 9.1.3).

\section{Results}

Keratin Expression Profiles in Mouse Liver, Common Bile Duct, Gallbladder, and Jejunum. The stoichiometric expression of keratin subtypes in mouse liver, gallbladder, and small intestine are known ${ }^{29,30}$; however, those of the common bile duct are not. We analyzed the keratin-enriched protein fractions from these tissues by Coomassie staining (Fig. 1A) and immunoblotting (Fig. 1B). As shown in Fig. 1, K8/K18/K19 are the major keratins in both gallbladder and common bile duct. In addition, the common bile duct, but not gallbladder, also expresses low levels of K7 and minimal amounts of K20, which can be detected by immune blotting (Fig. 1B) but not by Coomassie staining (Fig. 1A). In all tested organs, 
Table 1. Demographics of the PBC Patient Cohort

\begin{tabular}{|c|c|c|c|c|}
\hline & Total $n=201$ & Stage I-II $n=96$ & Stage III-IV $n=105$ & $P$ Value \\
\hline Female & $192(96 \%)$ & $93(97 \%)$ & 99 (94\%) & 0.72 \\
\hline Age at enrollment (years) & $53 \pm 12$ & $51 \pm 12$ & $55 \pm 12$ & 0.04 \\
\hline AMA-positive \# & $151(75 \%)$ & $73(76 \%)$ & $78(74 \%)$ & 0.74 \\
\hline Total bilirubin (mg/dL) & $0.87 \pm 0.70$ & $0.73 \pm 0.55$ & $1 \pm 0.80$ & 0.01 \\
\hline Albumin (g/dL) & $4.13 \pm 0.43$ & $4.16 \pm 0.42$ & $4.09 \pm 0.43$ & 0.24 \\
\hline Prothrombin time (INR) & $1.02 \pm 0.14$ & $1.01 \pm 0.16$ & $1.02 \pm 0.12$ & 0.42 \\
\hline Ascites (n) & 7 (3.5\%) & $1(1.04 \%)$ & $6(5.7 \%)$ & 0.08 \\
\hline Mayo score & $3.71 \pm 0.96$ & $3.49 \pm 0.74$ & $3.98 \pm 0.97$ & 0.0001 \\
\hline
\end{tabular}

The normal values of total bilirubin, albumin, and prothrombin time are $<1.0,>3.5$, and $<1.2$, respectively ( 8 patients lacked data for total bilirubin, albumin, and prothrombin time); INR, international normalized ratio; continuous variables are expressed as the mean \pm standard deviation; age was unknown in 17 patients; the $P$-value compares the patients with mild (stage I-II) and severe (stage III-IV) PBC.

A
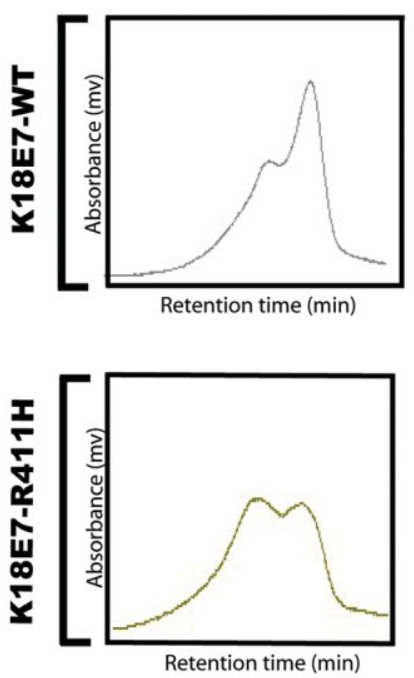

B
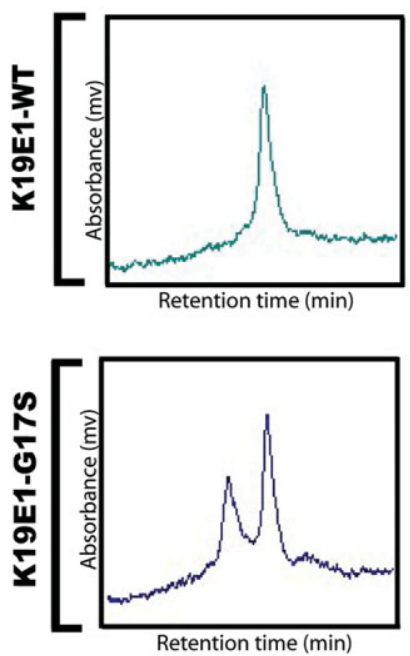

Forward

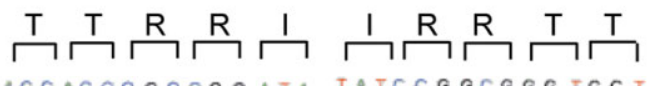

ACCACCCGCCGGATA TATCCGGCGGGTGGT
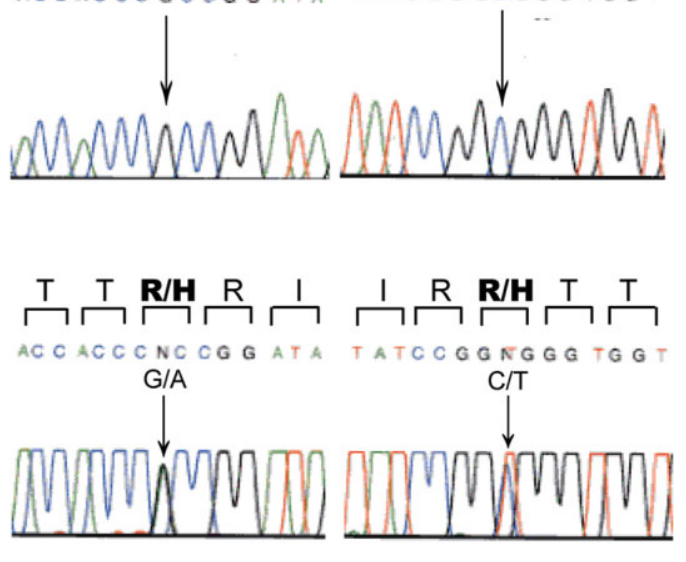

Forward

Reverse

否

T TCGGAGGCCTGGGC GCCCAGGCCTCCGAA

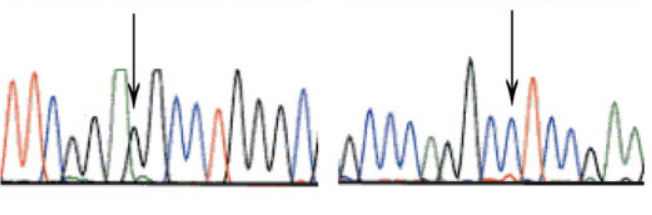

F G/S L G G

TTCGGRAGCCTGGGC GCCCAGGCTTCCGAA

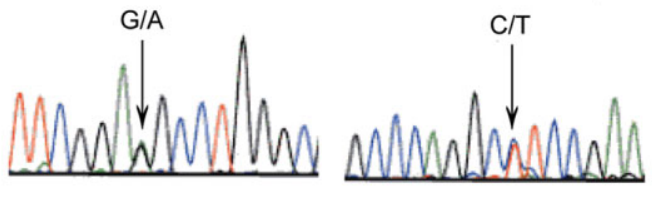

Fig. 2. Detection of keratin variants in patients with PBC and in blood bank donors. Genomic DNA was isolated from peripheral blood, and individual K18/K19 exonic regions were amplified by touchdown PCR then analyzed by DHPLC. Samples with a shifted chromatogram pattern were sequenced in the forward and reverse directions. The nucleotide locations of the variants were determined as described in "Patients and Methods." The novel heterozygous K18 exon 7-R411H (A) and K19 exon 1-G17S variants (B) are presented in comparison with their corresponding WT samples. 
Table 2. Keratin Variants in PBC and Control Groups

\begin{tabular}{|c|c|c|c|c|}
\hline \multirow[b]{2}{*}{ Keratin Gene } & \multicolumn{2}{|c|}{ Variants } & \multirow[b]{2}{*}{ PBC $(n=201)$} & \multirow[b]{2}{*}{ Control $(n=200)$} \\
\hline & Nucleotide & Amino Acid & & \\
\hline \multirow[t]{7}{*}{ KRT8 } & $184 \mathrm{G} \rightarrow \mathrm{T}$ & G62C & 4 & 0 \\
\hline & $1022 \mathrm{G} \rightarrow \mathrm{A}$ & $\mathrm{R} 341 \mathrm{H}$ & $8[8]$ & $\mathbf{3}[3]$ \\
\hline & $1138 \mathrm{G} \rightarrow \mathrm{A}$ & V380I & 1 & 0 \\
\hline & $187 \mathrm{~A} \rightarrow \mathrm{G}$ & I63V* & 1 & 0 \\
\hline & $681 \mathrm{~A} \rightarrow \mathrm{G}$ & L227L† & 28 & 32 \\
\hline & IVS6 $+46 \mathrm{~A} \rightarrow \mathrm{T} \ddagger$ & & 1 & 0 \\
\hline & IVS7 + 10 Del C & & $8[8]$ & $3[3]$ \\
\hline \multirow[t]{3}{*}{ KRT18 } & $1232 \mathrm{G} \rightarrow \mathrm{A}$ & R411H (new) & $1[1]$ & 0 \\
\hline & $689 \mathrm{G} \rightarrow \mathrm{C}$ & S230T* & $2[1]$ & 0 \\
\hline & IVS2 $+38 \mathrm{G} \rightarrow \mathrm{A} \ddagger$ & & $1[1]$ & 1 \\
\hline \multirow[t]{6}{*}{ KRT19 } & $49 \mathrm{G} \rightarrow \mathrm{A}$ & G17S (new) & 3 [1] & 0 \\
\hline & $685 \mathrm{G} \rightarrow \mathrm{T}$ & V229M (new) & 0 & 1 \\
\hline & $179 \mathrm{C} \rightarrow \mathrm{G}$ & A60G* & 19 & 20 \\
\hline & $90 \mathrm{C} \rightarrow \mathrm{T}$ & $\mathrm{A} 30 \mathrm{~A} \dagger$ & 18 & 18 \\
\hline & $471 \mathrm{~T} \rightarrow \mathrm{C}$ & $\mathrm{N} 157 \mathrm{~N} \dagger$ & 48 & 52 \\
\hline & $552 \mathrm{C} \rightarrow \mathrm{T}$ & N184N (new) $†$ & 1 & 0 \\
\hline Total & & & $17(8.5 \%)$ & $4(2 \%)$ \\
\hline
\end{tabular}

$P<0.004$ (when comparing the total number of biologically-relevant variants in patients with PBC versus controls); odds ratio $=4.5 ; 95 \%$ confidence interval $=$ 1.5-13.7. Amino acids are denoted by the single-letter abbreviations; "new" highlights novel variants that have not been previously reported; brackets indicate the number of compound variants.

*Highlights "polymorphisms" rather than "mutations" based on previous studies.

†Highlights "silent" nucleotide mutations that do not result in an amino acid change.

IHighlights intronic variants, which were assigned to their respective intervening sequence (IVS). Note that the IVS number refers to the neighboring $5^{\prime}$ exon. For example, IVS7 + 10 Del C depicts a noncoding deletion found 10 nucleotides downstream from the $3^{\prime}$-end of exon 7 in intervening sequence 7 . IVS6+ $45 \mathrm{~A} \rightarrow \mathrm{T}$ highlights a noncoding nucleotide substitution found 45 nucleotide downstream from the $3^{\prime}$-end of exon 6 in intervening sequence 6 .

K8 is the major type II keratin while K19 has limited expression in the liver (because of its absence in hepatocytes but presence in biliary epithelia).

Demographics and Characteristics of the PBC Patients. The demographics and clinical information of the PBC patients are summarized in Table 1 . A total of 201 patients with $\mathrm{PBC}$ were included in the final analysis because eight patient DNA samples were not reliably amplified. As expected, most patients were female (96\%). AMA was positive in 151 patients (75\%); 96 patients (48\%) exhibited early PBC stages I to II, and 105 patients (52\%) had advanced disease. Several factors including age, total bilirubin levels, and the Mayo score were significantly different in advanced versus early stages $(P<0.05)$.

Detection of Keratin Variants in PBC Patients. We analyzed all exonic regions of K8, K18, and K19 using DHPLC and subsequent DNA sequencing of the samples that displayed an abnormal elution profile off the $\mathrm{DH}$ PLC column. Of the 201 PBC patients, 17 (8.5\%) harbored five disease-associated heterozygous exonic variants including three $\mathrm{K} 8$ (G62/R341/V380I), one K18 (R411H) (Fig. 2A), and one K19 (G17S) (Fig. 2B). Keratin variants are defined as "disease-associated" (or biologically relevant) when they meet one or more of the following criteria: (1) are overrepresented in liver disease patients when compared with the appropriate control group, (2) alter a highly conserved amino acid, (3) introduce or ablate a charge or a posttranslational modification site such as phosphorylation, or (4) have been shown to cause a phenotype in transgenic mice. The variants K18 R411H and K19 G17S are newly described variants found in one and three patients with PBC, respectively, and were not detected in the control group (Table 2; Fig. 2). K18 R411 is not conserved among type I keratins but is conserved across human, mouse, and rat species, whereas K19 G17S is conserved among several type I keratins (not shown) and across several species (Supporting Fig. S1).

Some of the other variants we identified are considered polymorphisms rather than pathogenic mutations. This group included K8 I63V, K18 S230T, and K19 G60A, which were observed at comparable frequencies in the control and patient liver disease cohorts in previous studies. ${ }^{20,21}$ Four "silent" non-amino-acid-altering exonic variants (including the novel K19 N184N) and three intronic variants (including the novel K18 IVS2 + $38 \mathrm{G} \rightarrow \mathrm{A}$ found in one patient with $\mathrm{PBC}$ and in one control), which did not associate with hepatitis C-related liver disease progression, ${ }^{21}$ are also considered nonpathogenic (Table 2).

Among the disease-associated variants, K8 R341H was the most common (found in 8 of 17 PBC patients). Seven 
Table 3. Distribution of Disease-Associated Keratin Exonic Variants Among PBC Disease Stages, AMA-Positive and AMANegative, and ANA-Positive and ANA-Negative PBC Patients

\begin{tabular}{|c|c|c|c|c|c|c|c|c|}
\hline \multirow[b]{2}{*}{ Keratin Gene } & \multirow{2}{*}{\multicolumn{2}{|c|}{ Variants }} & \multicolumn{2}{|c|}{ Stage } & \multicolumn{2}{|c|}{ AMA } & \multicolumn{2}{|c|}{ ANA* } \\
\hline & & & $\begin{array}{l}\text { Stage I-II } \\
(\mathrm{n}=96)\end{array}$ & $\begin{array}{l}\text { Stage III-IV } \\
(n=105)\end{array}$ & $\begin{array}{l}\text { AMA Negative } \\
\quad(\mathbf{n}=\mathbf{5 0})\end{array}$ & $\begin{array}{l}\text { AMA Positive } \\
(\mathrm{n}=151)\end{array}$ & $\begin{array}{l}\text { ANA Negative } \\
\quad(n=77)\end{array}$ & $\begin{array}{l}\text { ANA Positive } \\
(\mathrm{n}=117)\end{array}$ \\
\hline & Nucleotide & Amino acid & & & & & & \\
\hline \multirow[t]{3}{*}{ KRT8 } & $184 \mathrm{G} \rightarrow \mathrm{T}$ & G62C & 0 & 4 & 0 & 4 & 2 & 1 \\
\hline & $1022 \mathrm{G} \rightarrow \mathrm{A}$ & $\mathrm{R} 341 \mathrm{H}$ & 3 & 5 & 2 & 6 & 3 & 5 \\
\hline & $1138 \mathrm{G} \rightarrow \mathrm{A}$ & V380I & 0 & 1 & 0 & 1 & 1 & 0 \\
\hline KRT18 & $1232 \mathrm{G} \rightarrow \mathrm{A}$ & R411H (new) & 1 & 0 & 0 & 1 & 1 & 0 \\
\hline KRT19 & $49 \mathrm{G} \rightarrow \mathrm{A}$ & G17S (new) & 0 & 3 & 1 & 2 & 0 & 3 \\
\hline Total & & & $4(4.2 \%)$ & 13 (12.4\%) & $3(6.0 \%)$ & 14 (9.3\%) & 7 (9.0\%) & $9(7.7 \%)$ \\
\hline$P$ (odds ratio, $95 \%$ confidence interval) & & & \multicolumn{2}{|c|}{0.04} & \multicolumn{2}{|c|}{0.47} & \multicolumn{2}{|c|}{$\begin{array}{c}0.73 \\
(1.2,0.42-3.37)\end{array}$} \\
\hline
\end{tabular}

Amino acids are denoted by the single-letter abbreviations. "New" highlights novel variants that have not been previously reported.

* Seven cases (including one harboring a $\mathrm{K} 862 \mathrm{C}$ variant) were excluded from analysis because of missing ANA values.

patients had single and 10 had compound heterozygous variants, including one K18 R411H+K18 S230T, one $\mathrm{K} 19 \mathrm{G} 17 \mathrm{~S}+\mathrm{K} 18-\mathrm{IVS} 2+38 \mathrm{G} \rightarrow \mathrm{A}$, and the eight $\mathrm{K} 8$ $\mathrm{R} 341 \mathrm{H}$ carriers who concurrently carried the K8 IVS7+10delC polymorphism. The exclusive association between K8 R341H and K8 IVS7+10delC, which was noted previously, ${ }^{21,23}$ is also present in the control samples.

In the control group, the frequency of disease-associated exonic variants was 2\% (4 of 200; that includes K8 $\mathrm{R} 341 \mathrm{H}$ and $\mathrm{K} 19 \mathrm{~V} 229 \mathrm{M})$. The K8 R341H variant was observed in three subjects, whereas one donor carried a novel K19 V229M variant (Table 2), which is conserved among species and among several other type I keratins (Supporting Fig. S1). Notably, there is a significantly higher frequency of disease-associated/biologically relevant keratin variants in $\mathrm{PBC}$ patients (highlighted in bold lettering in Table 2$)$ versus the control groups $(P<0.004$, odds ratio $=4.5,95 \%$ confidence interval $=1.5-13.7)$.

Relationship of Keratin Variants with PBC. Of the 17 patients who harbored relevant keratin variants, four were seen in 96 patients with early disease stage (4.2\%), whereas 13 were found in 105 subjects with advanced disease (12.4\%; Table 3). The frequency of keratin variants in the advanced stage of $\mathrm{PBC}$ was significantly higher than in the early stage $(P<0.04$, odds ratio $=3.25,95 \%$ confidence interval $=1.02-10.4)$, thereby suggesting that keratin variants associate with liver disease progression in PBC. Conversely, there was no association between keratin variants and the presence of AMA, because 14 of 151 AMA-positive patients (9.3\%) had relevant keratin variants as compared with 3 of 50 (6\%) AMA-negative patients $(P=0.47)$ (Table 3$)$. The frequency of keratin variants among antinuclear antibody-negative and antinuclear antibody-positive PBC patients was also similar (Table 3). The 17 relevant keratin variants were found at similar frequencies in both sexes, and patients carrying the variants did not differ from the overall group with respect to their bilirubin and albumin serum levels, prothrombin time, Mayo score, or the presence of ascites (Table 4). We were not able to include analysis of the estimated duration of disease in those with and without the keratin variants because of incomplete data on cholestasis parameters before the diagnosis of PBC.

\section{Discussion}

The Importance of Keratin Variants in Human Liver Disease. Our results show that keratin variants are overrepresented in Italian patients with PBC when compared with blood bank donors $(8.5 \%$ versus $2 \% ; P<$ 0.004 , odds ratio $=4.5,95 \%$, confidence interval $=$ 1.5-13.7). The frequency of keratin variants presented here is somewhat lower that seen in a US cohort of explants from patients with end-stage liver diseases of multiple origins (12.4\% in liver explants versus $3.7 \%$ in blood bank controls ${ }^{20}$ but similar to that observed in a German

Table 4. The Clinical Features of PBC Patients Harboring Significant Keratin Variants

\begin{tabular}{lccc}
\hline & \multicolumn{2}{c}{ Presence of Keratin Variants } & \\
\cline { 2 - 3 } & Yes $\mathbf{( n = 1 7 )}$ & No $(\mathbf{n}=\mathbf{1 8 4})$ & P Value \\
\hline Sex (Male/Female) & $1 / 16$ & $8 / 176$ & 0.77 \\
Age at enrollment (years) & $55 \pm 13$ & $53 \pm 12$ & 0.59 \\
Total billirubin (mg/dL) & $0.70 \pm 0.28$ & $0.88 \pm 0.73$ & 0.30 \\
Albumin (g/dL) & $4.25 \pm 0.32$ & $4.11 \pm 0.44$ & 0.22 \\
Prothrombin time (INR) & $1.00 \pm 0.13$ & $1.02 \pm 0.14$ & 0.56 \\
Ascites (yes/no) & $1 / 16$ & $5 / 171$ & 0.53 \\
Mayo score & $3.65 \pm 0.20$ & $3.82 \pm 0.79$ & 0.54 \\
\hline
\end{tabular}

Normal values of total bilirubin, albumin, and prothrombin time are $<1.0$, $>3.5$, and $<1.2$, respectively; INR, international normalized ratio; Continuous variables are expressed as the mean \pm standard deviation. 

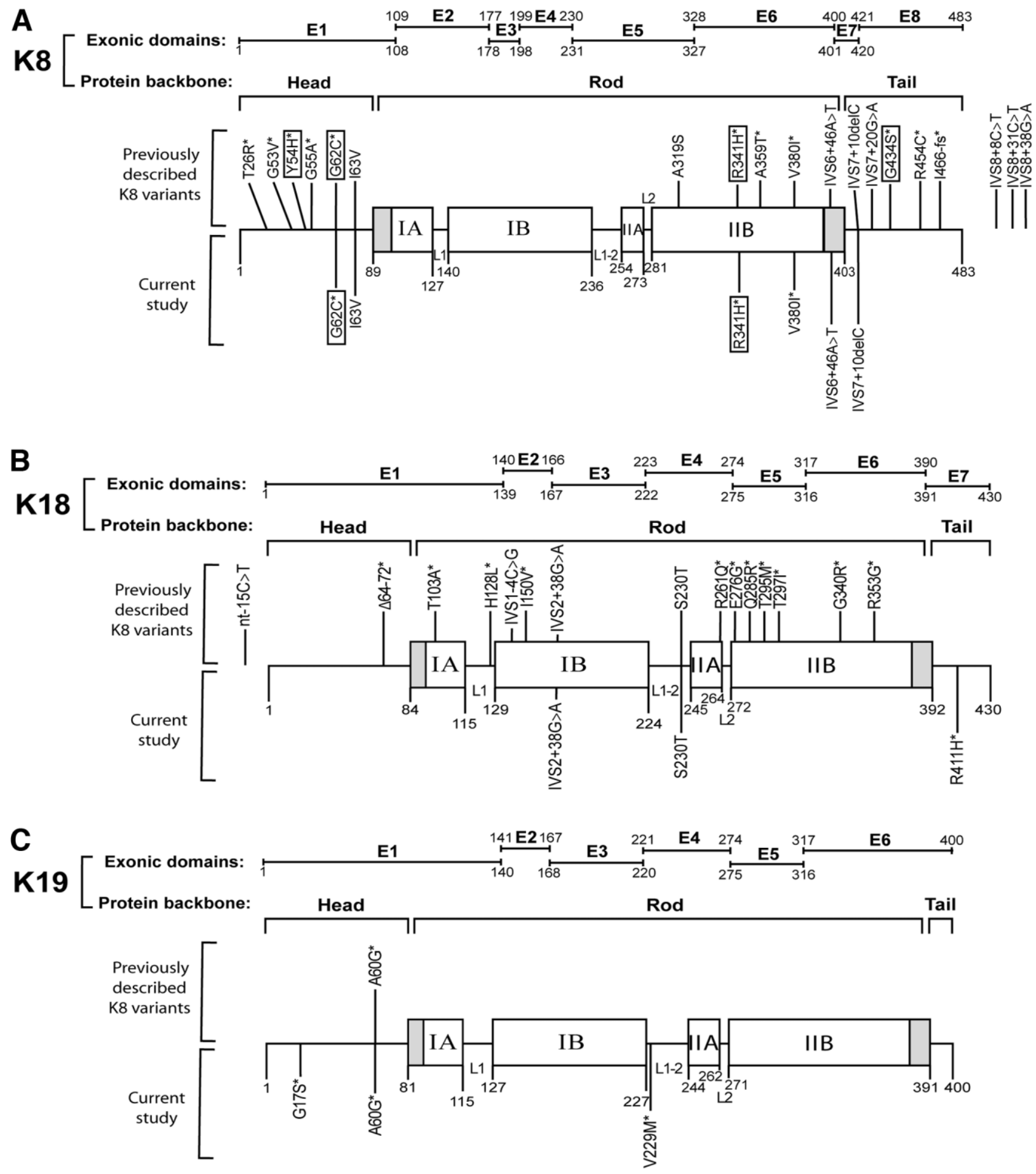

Fig. 3. Distribution of K8/K18/K19 variants within the keratin backbone. The schematic shows K8 (A), K18 (B), and K19 (C) exonic regions together with their respective protein domains. Keratins exhibit a tripartite structure consisting of a central $\alpha$-helical "rod" as well as non- $\alpha$-helical $\mathrm{N}$-terminal "head" and C-terminal "tail" domains. The rod domain is subdivided into IA, IB, IIA, and IIB parts divided by nonhelical linker (L1, L1-2, and $L 2$ ) sequences. The shaded regions at the beginning and end of rod domains are highly conserved and represent mutation "hot spots" in epidermal keratins and other IF proteins. However, no human K8, K18, or K19 mutations have been identified in these hot spots. The K8/K18/K19 variants identified previously are displayed above the keratin protein backbone, whereas the variants described in this study are drawn below the protein backbone. Amino acids are represented by single-letter abbreviations. The most frequent K8 variants are boxed, whereas unmarked variants are seen at lower frequencies. The likely biologically significant variants are highlighted by an asterisk. K18 R411H and K19 G17S are the two novel variants identified in PBC patients, whereas K19 V229M was observed only in one healthy blood bank donor. The intervening sequence (IVS) number refers to the neighboring $5^{\prime}$ exon. For example, IVS7 +10 Del C depicts a noncoding deletion found 10 nucleotides downstream from the $3^{\prime}$-end of exon 7 in intervening sequence $7 . \mathrm{E}$, exon; nt, nucleotides. 
cohort with chronic hepatitis $\mathrm{C}$ virus infection $(7.8 \%) .^{21}$ In addition, the distribution of K8/K18/K19 variants within the keratin protein backbone (Fig. 3) mimics the previously published data and highlights several points. First, K8/K18/K19 variants are not found in the helix initiation and termination motifs of the rod domains of K8/K18/K19 (shaded areas at the beginning and end of the rod domain, Fig. 3), which are the regions where most epidermal keratin mutations cluster. ${ }^{31,32}$ This provides a likely explanation as to why K8/K18/K19 mutations pose a predisposing risk rather than cause disease per se. Second, most variants are rare ( $<1 \%$ frequency), which leads us to pool their frequencies when carrying out power analysis. Third, K8 has the most keratin variants, as compared with $\mathrm{K} 18$ and K19. K8 is the major keratin in hepatobiliary epithelial cells as observed in mice (Fig. 1) and, presumably, also in humans, although this has not been tested quantitatively. For K18, the novel $\mathrm{R} 411 \mathrm{H}$ variant identified herein is the first $\mathrm{K} 18$ tail domain variant described.

Genetic Risk Factors for Human PBC. Genetic factors are likely to play an important role in $\mathrm{PBC}$ pathogenesis. For example, family members of patients with $\mathrm{PBC}$ have a higher risk of developing the disease, with a concordance rate as high as $63 \%$ in monozygotic twins, ${ }^{8}$ which is among the highest reported in autoimmune diseases. Moreover, PBC predominance in females may relate to the higher incidence of X-chromosome monosomy in lymphoid cells. ${ }^{33}$ Several candidate genes and chromosomal loci have been described, but the data are inconclusive and at times contradictory. ${ }^{1,10-12}$ In addition, the focus has been primarily on proinflammatory and immunoregulatory-related genes, such as cytotoxic T lymphocyte antigen-4, interleukins, T helper 1 cells, or 1,25-dihydroxyvitamin d receptor., ${ }^{3,10-12}$ Therefore, the findings herein provide for the first time a potential link between the keratin cytoskeleton and autoimmune liver disease, possibly also contributing to the high tissue specificity of PBC. To our knowledge, no such association has been described between IF genes and immune response modulation, although leukocytes that lack vimentin have a reduced capacity to home to mesenteric lymph nodes and vimentinnull endothelial cells have a compromised integrity. ${ }^{34} \mathrm{In}$ addition, K8-null mice develop spontaneous Th2-type colitis. ${ }^{35,36}$ It remains to be determined how K8/K18/K19 mutations may predispose to autoimmune liver disease. One hypothesis is that keratin mutation-induced susceptibility to hepatocyte/cholangiocyte injury and death over time results in an autoimmune disease because of release of potential autoantigens in a susceptible host. In support of this, K8-null mice develop serum AMA though they do not develop autoimmune liver disease per se (unpublished observations). Given the expression of K8/K18/K19 in a variety of epithe- lial tissues, the keratin variant-based predisposition to $\mathrm{PBC}$ (and potentially other autoimmune diseases) may also be liver independent. For example, K8/K18/K19 are found in a subset of thymic epithelial cells, ${ }^{37}$ and proper keratin architecture is crucial for thymic microenvironment, as seen in mice ectopically expressing K10 in thymic cells that develop severe thymic alterations. ${ }^{38}$

K19 Joins the Ranks of Other Disease-Associated Keratins. Our study identified three K19 G17S variants among the 201 PBC patients analyzed. Of note, the K19 G17S variant was not found in the 200 Italian control blood bank donors or in a previous study that analyzed 200 US patients with inflammatory bowel disease and 70 US control subjects $(P=0.03$ for comparison of $\mathrm{PBC}$ versus the pooled Italian and US non-PBC subjects). ${ }^{23}$ Therefore, the K19 G17S variant is significantly overrepresented in PBC patients, which adds K19 to the list of 16 epithelial keratins, whose variants are known to cause or predispose to the development of human disease. ${ }^{39,40}$ However, because K19 variants are rare, larger studies are needed to confirm these findings. In addition, further studies using K19-null mice are warranted to characterize the impact of K19 deficiency on liver injury. One study did not show an obvious liver phenotype in K19-null mice, ${ }^{29}$ whereas a mild skeletal myopathy with a complex disorganization of muscular architecture was observed in these animals. ${ }^{41}$ The lack of an obvious disease phenotype in epithelial tissues of K19-null mice is likely to be related to the functional redundancy imparted by the expression of other type I keratins (such as K18 and K20).30,42

Acknowledgment: The authors thank all the patients and other donors who have made it possible to carry out the genetic analyses.

\section{References}

1. Kaplan MM, Gershwin ME. Primary biliary cirrhosis. N Engl J Med 2005;353:1261-1273.

2. Talwalkar JA, Lindor KD. Primary biliary cirrhosis. Lancet 2003;362:5361.

3. Selmi C, Invernizzi P, Zuin M, Podda M, Seldin MF, Gershwin ME. Genes and (auto)immunity in primary biliary cirrhosis. Genes Immun 2005;6:543-556.

4. Lazaridis KN, Talwalkar JA. Clinical epidemiology of primary biliary cirrhosis: Incidence, prevalence, and impact of therapy. J Clin Gastroenterol 2007;41:494-500.

5. Gross RG, Odin JA. Recent advances in the epidemiology of primary biliary cirrhosis. Clin Liver Dis 2008;12:289-303.

6. Mendes FD, Kim WR, Pedersen R, Therneau T, Lindor KD. Mortality attributable to cholestatic liver disease in the United States. HepatologY 2008;47:1241-1247.

7. Bittencourt PL, Farias AQ, Abrantes-Lemos CP, Goncalves LL, Goncalves PL, Magalhaes EP, et al. Prevalence of immune disturbances and chronic liver disease in family members of patients with primary biliary cirrhosis. J Gastroenterol Hepatol 2004;19:873-878. 
8. Selmi C, Mayo MJ, Bach N, Ishibashi H, Invernizzi P, Gish RG, et al. Primary biliary cirrhosis in monozygotic and dizygotic twins: genetics, epigenetics, and environment. Gastroenterology 2004;127:485-492.

9. Gershwin ME, Selmi C, Worman HJ, Gold EB, Watnik M, Utts J, et al. Risk factors and comorbidities in primary biliary cirrhosis: a controlled interview-based study of 1032 patients. Hepatology 2005;42:11941202.

10. Jones DE, Donaldson PT. Genetic factors in the pathogenesis of primary biliary cirrhosis. Clin Liver Dis 2003;7:841-864.

11. Osterreicher $\mathrm{CH}$, Stickel F, Brenner DA. Genomics of liver fibrosis and cirrhosis. Semin Liver Dis 2007;27:28-43.

12. Juran BD, Lazaridis KN. Genetics and genomics of primary biliary cirrhosis. Clin Liver Dis 2008;12:349-365.

13. Coulombe PA, Omary MB. 'Hard' and 'soft' principles defining the structure, function and regulation of keratin intermediate filaments. Curr Opin Cell Biol 2002;14:110-122.

14. Schweizer J, Bowden PE, Coulombe PA, Langbein L, Lane EB, Magin TM, et al. New consensus nomenclature for mammalian keratins. J Cell Biol 2006;174:169-174

15. Omary MB, Ku NO, Strnad P, Hanada S. Towards unraveling the complexity of 'simple' epithelial keratins in human disease. J Clin Invest 2009; in press.

16. Baribault H, Price J, Miyai K, Oshima RG. Mid-gestational lethality in mice lacking keratin 8. Genes Dev 1993;7:1191-1202.

17. Loranger A, Duclos S, Grenier A, Price J, Wilson-Heiner M, Baribault H, et al. Simple epithelium keratins are required for maintenance of hepatocyte integrity. Am J Pathol 1997;151:1673-1683.

18. Ku NO, Michie S, Oshima RG, Omary MB. Chronic hepatitis, hepatocyte fragility, and increased soluble phosphoglycokeratins in transgenic mice expressing a keratin 18 conserved arginine mutant. J Cell Biol 1995; 131:1303-1314.

19. Ku NO, Gish R, Wright TL, Omary MB. Keratin 8 mutations in patients with cryptogenic liver disease. N Engl J Med 2001;344:1580-1587.

20. Ku NO, Lim JK, Krams SM, Esquivel CO, Keeffe EB, Wright TL, et al. Keratins as susceptibility genes for end-stage liver disease. Gastroenterology 2005;129:885-893.

21. Strnad P, Lienau TC, Tao GZ, Lazzeroni LC, Stickel F, Schuppan D, et al. Keratin variants associate with progression of fibrosis during chronic hepatitis C infection. Hepatology 2006;43:1354-1363.

22. Ku NO, Omary MB. A disease- and phosphorylation-related nonmechanical function for keratin 8. J Cell Biol 2006;174:115-125.

23. Tao GZ, Strnad P, Zhou Q, Kamal A, Zhang L, Madani ND, et al. Analysis of keratin polypeptides 8 and 19 variants in inflammatory bowel disease. Clin Gastroenterol Hepatol 2007;5:857-864.

24. Ku NO, Toivola DM, Zhou Q, Tao GZ, Zhong B, Omary MB. Studying simple epithelial keratins in cells and tissues. Methods Cell Biol 2004;8: 489-517.

25. Zhong B, Zhou Q, Toivola DM, Tao GZ, Resurreccion EZ, Omary MB. Organ-specific stress induces mouse pancreatic keratin overexpression in association with NF-kappaB activation. J Cell Sci 2004;117:1709-1719.
26. Ludwig J, Dickson ER, McDonald GSA. Staging of chronic nonsuppurative destructive cholangitis (syndrome of primary biliary cirrhosis). Virchows Arch A Pathol Anat Histol 1978;379:103-112.

27. Dickson ER, Grambsch PM, Fleming TR, Fisher LD, Langangworthy A. Prognosis in primary biliary cirrhosis: model for decision making. HePATOLOGY 1989;10:1-7.

28. Strnad P, Lienau TC, Tao GZ, Ku NO, Magin TM, Omary MB. Denaturing temperature selection may underestimate keratin mutation detection by DHPLC. Hum Mutat 2006;27:444-452.

29. Tao GZ, Toivola DM, Zhong B, Michie SA, Resurreccion EZ, Tamai Y, et al. Keratin-8 null mice have different gallbladder and liver susceptibility to lithogenic diet-induced injury. J Cell Sci 2003;116:4629-4638.

30. Zhou Q, Toivola DM, Feng N, Greenberg HB, Franke WW, Omary MB. Keratin 20 helps maintain intermediate filament organization in intestinal epithelia. Mol Biol Cell 2003;14:2959-2971.

31. Ku NO, Strnad P, Zhang BH, Tao GZ, Omary MB. Keratins let liver live: mutations predispose to liver disease and crosslinking generates MalloryDenk bodies. Hepatology 2007;46:1639-1649.

32. Uitto J, Richard G, McGrath JA. Diseases of epidermal keratins and their linker proteins. Exp Cell Res 2007;313:1995-2009.

33. Invernizzi P, Miozzo M, Battezzati PM, Bianchi I, Grati FR, Simoni G, et al. Frequency of monosomy $\mathrm{X}$ in women with primary biliary cirrhosis. Lancet 2004;363:533-535.

34. Nieminen M, Henttinen T, Merinen M, Marttila-Ichihara F, Eriksson JE, Jalkanen S. Vimentin function in lymphocyte adhesion and transcellular migration. Nat Cell Biol 2006;8:156-162.

35. Baribault H, Penner J, Iozzo RV, Wilson-Heiner M. Colorectal hyperplasia and inflammation in keratin 8-deficient FVB/N mice. Genes Dev 1994; 8:2964-2973.

36. Habtezion A, Toivola DM, Butcher EC, Omary MB. Keratin-8-deficient mice develop chronic spontaneous $\mathrm{Th} 2$ colitis amenable to antibiotic treatment. J Cell Sci 2005;118:1971-1980.

37. Savino W, Dardenne M. Developmental studies on expression of monoclonal antibody-defined cytokeratins by thymic epithelial cells from normal and autoimmune mice. J Histochem Cytochem 1988;36:1123-1129.

38. Santos M, Río P, Ruiz S, Martínez-Palacio J, Segrelles C, Lara MF, Segovia JC, et al. Altered T cell differentiation and Notch signaling induced by the ectopic expression of keratin K10 in the epithelial cells of the thymus. J Cell Biochem 2005;95:543-558

39. Omary MB, Coulombe PA, McLean WH. Intermediate filament proteins and their associated diseases. N Engl J Med 2004;351:2087-2100.

40. Szeverenyi I, Cassidy AJ, Chung CW, Lee BT, Common JE, Ogg SC, et al. The Human Intermediate Filament Database: comprehensive information on a gene family involved in many human diseases. Hum Mutat 2008;29: 351-360.

41. Stone MR, O’Neill A, Lovering RM, Strong J, Resneck WG, Reed PW, et al. Absence of keratin 19 in mice causes skeletal myopathy with mitochondrial and sarcolemmal reorganization. J Cell Sci 2007;120:3999-4008.

42. Hesse M, Grund C, Herrmann H, Bröhl D, Franz T, Omary MB, et al. A mutation of keratin 18 within the coil $1 \mathrm{~A}$ consensus motif causes widespread keratin aggregation but cell type-restricted lethality in mice. Exp Cell Res 2007:313:3127-3140. 